Review

\title{
Recent Advances in Electrochemical Sensing of Isoproterenol
}

\author{
Sayed Zia Mohammadi ${ }^{1}$, Hadi Beitollahi ${ }^{2, *}$, Mohadeseh Safaei ${ }^{2}$, Quyet Van Le ${ }^{3, *}$, Ho Won Jang ${ }^{4}$, \\ Mohammadreza Shokouhimehr ${ }^{4, *}$, Wanxi Peng ${ }^{5,6, *}$ \\ ${ }^{1}$ Department of Chemistry, Payame Noor University, Tehran, Iran. \\ ${ }^{2}$ Environment Department, Institute of Science and High Technology and Environmental Sciences, \\ Graduate University of Advanced Technology, Kerman, Iran. \\ ${ }^{3}$ Institute of Research and Development, Duy Tan University, Da Nang 550000, Vietnam. \\ ${ }^{4}$ Department of Materials Science and Engineering, Research Institute of Advanced Materials, Seoul \\ National University, Seoul 08826, Republic of Korea. \\ ${ }^{5}$ College of Forestry, Henan Agricultural University, Zhengzhou 450002, China. \\ ${ }^{6}$ School of Automotive Engineering, Huanghe Jiaotong University, Jiaozuo 454950, China. \\ *E-mail: h.beitollahi@yahoo.com (H.B), Levanquyet@dtu.edu.vn (Q.V.L.); mrsh2@snu.ac.kr (M.S.) \\ pengwanxi@163.com (W.P.)
}

doi: $10.20964 / 2021.05 .34$

Received: 24 June 2020 / Accepted: 30 July 2020 / Published: 31 March 2021

One of the sympathomimetic beta-adrenergic agonist medications is isoproterenol (IP), which is utilized for the treatment of heart block or bradycardia. It applies positive chrono-tropic and inotropic impacts on heart in case of heart attack, bronchitis, and cardiac chock. However, its excessive dosage may result in the arrhythmias or heart failure. Consequently, it is necessary to devise simplistic, fast, and affordable assays (e.g., electro-chemical determination) for aiding the clinical diagnostic and treatments of IP. In this review, we have covered important techniques and electrode substances utilized for the IP analysis base on the nanostructured electrodes and their chemically modifications. In addition, we have illustrated the advantages and disadvantages of the presented the electrode materials for the IP analyses.

Keywords: Isoproterenol, drug, Clinical diagnostics, Electrochemical detection, Chemically modified Electrodes.

\section{FULL TEXT}

(C) 2021 The Authors. Published by ESG (www.electrochemsci.org). This article is an open access article distributed under the terms and conditions of the Creative Commons Attribution license (http://creativecommons.org/licenses/by/4.0/). 